\title{
The genus crossing number
}

\author{
Bojan Mohar * \\ Department of Mathematics, Simon Fraser University \\ Burnaby, B.C. V5A 1S6, Canada
}

Received 1 August 2007, accepted 11 July 2009, published online 26 September 2009

\begin{abstract}
Pach and Tóth [5] introduced a new version of the crossing number parameter, called the degenerate crossing number, by considering proper drawings of a graph in the plane and counting multiple crossing of edges through the same point as a single crossing when all pairwise crossings of edges at that point are transversal. We propose a related parameter, called the genus crossing number, where edges in the drawing need not be represented by simple arcs. This relaxation has two important advantages. First, the genus crossing number is invariant under taking subdivisions of edges and is also a minor-monotone graph invariant. Secondly, it is "computable" in many instances, which is a rare phenomenon in the theory of crossing numbers. These facts follow from the proof that the genus crossing number is indeed equal to the non-orientable genus of the graph. It remains an open question if the genus crossing number can be strictly smaller than the degenerate crossing number of Pach and Tóth. A relation to the minor crossing number introduced by Bokal, Fijavž, and Mohar [1] is also discussed.
\end{abstract}

Keywords: Crossing number, genus.

Math. Subj. Class.: 05C10

\section{Introduction}

Let $G$ be a graph. The crossing number of $G$, denoted by $\mathrm{CR}(G)$, is the minimum number of edge-crossings taken over all proper drawings of $G$ in the plane. By a drawing we refer to a representation of the graph in the Euclidean plane, where vertices are points in the plane and edges are piecewise linear arcs joining their end-vertices. A drawing is proper if edges are simple arcs and their interiors do not contain vertices of the graph. If $k$ edges intersect at the same point (not a vertex), then each of the $\left(\begin{array}{l}k \\ 2\end{array}\right)$ pairwise crossings counts

* Supported in part by the ARRS, Research Program P1-0507, and by an NSERC Discovery Grant. On leave from Department of Mathematics, FMF \& IMFM, University of Ljubljana, 1000 Ljubljana, Slovenia.

E-mail address: mohar@sfu.ca (Bojan Mohar) 
towards the computation of the total number of crossings of the drawing when we consider $\mathrm{CR}(G)$.

Pach and Tóth [5] introduced a modified definition of the crossing number of a graph $G$, called the degenerate crossing number and denoted by $\mathrm{CR}(G)$, where a multiple crossing at a point $p$ in the plane is counted as a single crossing if all pairs of edges passing through $p$ intersect transversally, i.e., they locally intersect at $p$ and the intersection is not tangential. Such a point $p$ will be referred to as a crossing point. The initial goal to introduce this notion was to investigate to what extent does this modification affect the value of the crossing number.

Pach and Tóth [5] also introduced the notion of a crossing number, denoted by $\mathrm{CR}^{*}(G)$, where multiple, pairwise transversal crossing at the same point is counted as a single crossing, but two distinct edges are allowed to cross each other at most once. While $\mathrm{CR}^{*}(G) \geq \underline{\mathrm{CR}}(G)$ holds for any graph $G$, it is proved in [5] that $\mathrm{CR}^{*}(G)$ can be much bigger than $\mathrm{CR}(G)$, thus showing that multiple crossings between distinct edges may help reducing the corresponding value of the crossing number.

The purpose of this paper is to propose another modification, where we do not insist that edges are simple arcs. We allow self-crossings of arcs representing the edges, but all self-crossings should be transversal. For reasons that will become apparent soon, we call the minimum number of crossing points of such drawings of $G$ the genus crossing number of $G$ and denote it by $\operatorname{GCR}(G)$. It is clear that

$$
\operatorname{GCR}(G) \leq \underline{\mathrm{CR}}(G) \leq \mathrm{CR}^{*}(G) \leq \mathrm{CR}(G) .
$$

It is an open question if $\operatorname{GCR}(G)$ can be strictly smaller than $\underline{\mathrm{CR}}(G)$. We shall discuss this issue in Section 3. Other inequalities in (1.1) can be strict inequalities, see [5].

The newly introduced version of the crossing number has some advantages over other notions of the crossing number mentioned above or treated elsewhere in the literature, see, e.g. [4, 6]:

(1) The genus crossing number is the first crossing number invariant, for which exact values can be determined for important families of graphs, like the complete or complete multipartite graphs, the hypercubes, etc. See Section 2 for details. Despite this fact, it is still NP-hard to compute the genus crossing number for arbitrary graphs.

(2) The genus crossing number is minor-monotone, i.e., if a graph $H$ is a minor of $G$, then $\operatorname{GCR}(H) \leq \operatorname{GCR}(G)$.

(3) Unlike $\underline{\mathrm{CR}}$ and $\mathrm{CR}^{*}$, the genus crossing number is a topological invariant, i.e., it does not change if we subdivide edges. Moreover, if we subdivide each edge of $G$ by inserting sufficiently many vertices of degree 2 , then we can get rid of all multiple crossings and all self-crossings of edges with respect to some optimal drawing of $G$. Consequently, the resulting subdivision $S$ satisfies

$$
\mathrm{CR}^{*}(G) \geq \underline{\mathrm{CR}}(G) \geq \mathrm{CR}^{*}(S)=\underline{\mathrm{CR}}(S)=\operatorname{GCR}(S)=\operatorname{GCR}(G) .
$$

\section{The genus crossing number}

As stated in the introduction, we shall consider the version of the crossing number where edges need not be simple Jordan curves. They are allowed to self-intersect and also two distinct edges are allowed to intersect more than once. However, interiors of edges should not 
contain vertices of the graph and all intersections should be transversal, i.e., non-tangential. In particular, if edges cross at a point $p$, possibly some edges passing through $p$ more than once, we require that all passes through $p$ are transversal to each other. Now we define $\operatorname{GCR}(G)$ as the minimum integer $k$ such that $G$ has a drawing in the plane with the above properties and with $k$ points where distinct edges cross each other.

Recall that the nonorientable genus $\tilde{\mathbf{g}}(G)$ of a graph $G$ is the minimum genus of a nonorientable surface in which $G$ can be embedded, cf. [3]. Having a drawing of $G$ in the plane with $g=\operatorname{GCR}(G)$ points at which the edges cross, each such crossing can be replaced by a crosscap, yielding an embedding of $G$ into the non-orientable surface of genus $g$ (if $g>0$ ). This shows that $\operatorname{GCR}(G) \geq \tilde{\mathbf{g}}(G)$ if $G$ is nonplanar. It is interesting that the converse also holds:

Theorem 2.1. Let $G$ be a non-planar graph. Then $\operatorname{GCR}(G)=\tilde{\mathbf{g}}(G)$.

Proof. Let $g=\tilde{\mathbf{g}}(G)$, and let us consider an embedding of $G$ in the non-orientable surface $S$ of genus $g$. Since $S$ is homeomorphic to the connected sum of $g$ projective planes, it contains $g$ pairwise disjoint 1 -sided simple closed curves $\alpha_{1}, \ldots, \alpha_{g}$. We may assume that none of these curves passes through a vertex of $G$ and that each of them intersects $G$ in finitely many points. By cutting the surface along all curves $\alpha_{1}, \ldots, \alpha_{g}$, we obtain the surface homeomorphic to the sphere with $g$ holes. By contracting each of these holes to a point, a drawing of $G$ in the plane is obtained. It is easy to see that each contracted hole gives rise to a (multiple) crossing, in which all edges cross transversally. This shows that $\operatorname{GCR}(G) \leq g=\tilde{\mathbf{g}}(G)$.

Known results about the nonorientable genus of graphs (see, e.g., [2, 3, 8]) yield exact values for all most common families of graphs, in particular for the complete and complete bipartite graphs:

Corollary 2.2. For $n \geq 8$ and for $k \geq l \geq 3$, we have:

$$
\operatorname{GCR}\left(K_{n}\right)=\left\lceil\frac{(n-3)(n-4)}{6}\right\rceil \text { and } \operatorname{GCR}\left(K_{k, l}\right)=\left\lceil\frac{(k-2)(l-2)}{2}\right\rceil .
$$

We refer to monographs on topological graph theory $[2,3,8]$ for many other exact results about the non-orientable genus, and hence also for the genus crossing number of graphs. Theorem 2.1 thus enables us to compute or estimate the genus crossing number for most important families of graphs, like the complete or complete multipartite graphs, the hypercubes, etc. In that sense, GCR is the first crossing number invariant, for which exact values can be determined for so many graphs.

Nevertheless, it is still hard to compute the genus crossing number for arbitrary graphs. This follows from Thomassen's results in [7], where it is shown that it is NP-hard to compute the non-orientable genus.

Corollary 2.3. It is NP-hard to determine the genus crossing number.

There are other consequences of Theorem 2.1. For instance, it is clear that GCR is a minor-monotone graph parameter. This issue is treated in more details in Section 4. Another easy consequence are upper and lower bounds on $\operatorname{GCR}(G)$. 
Theorem 2.4. Let $G$ be a connected graph of order $n \geq 2$ and with $e$ edges, and let $g$ be the girth of $G$. Let $r$ be the maximum number of edges in an outerplanar subgraph of $G$. Then

$$
\frac{g-2}{g} e-n+2 \leq \operatorname{GCR}(G) \leq \begin{cases}e-r-1 & \text { if } r<e, \\ 0 & \text { if } r=e .\end{cases}
$$

Proof. If $G$ is not simple, then $g \leq 2$, and the lower bound is trivial. Since adding loops and parallel edges has no effect on $\operatorname{GCR}(G)$ and does not decrease the value of $e-r-1$, we may henceforth assume that $G$ is a simple graph. Let $c=\operatorname{GCR}(G)$, and let us consider an embedding of $G$ in $\mathbb{N}_{c}$. Let $f$ be the number of facial walks. The girth condition shows that $f \leq 2 e / g$. Now, Euler's formula implies that $c \geq 2-n+e-f \geq 2-n+(g-2) e / g$. This yields the lower bound.

To prove the upper bound, let $H$ be a maximal outerplanar graph of $G$. Consider an embedding of $H$ in the plane so that all vertices of $G$ are on the boundary of the outer face. Clearly, $H$ is a spanning subgraph of $G$. Now let us define an embedding of $G$ as follows. (Cf. [3] for details about combinatorial description of 2-cell embeddings using rotation systems and signatures.) First, define an arbitrary rotation system for $G$ so that all bounded faces of $H$ remain facial walks with respect to the extended embedding. Finally, if $|E(G) \backslash E(H)|=e-r>0$, set the signature on one edge $e_{0} \in E(G) \backslash E(H)$ to be positive, and set it negative for all other edges in $E(G) \backslash E(H)$. By extending the embedding of $H$ edge-by-edge, $e_{0}$ being the last one to be added, we see that in all partial embeddings obtained during this process, the outer face of $H$ is replaced by a single facial walk. This means that each added edge decreases the Euler characteristic of the resulting surface by 1 . On the other hand, the last edge $e_{0}$ divides the outer facial walk into two, and hence does not change the Euler characteristic. Thus, the nonorientable genus of the resulting surface (if $e-r \geq 2$ ) is equal to $e-r-1$. If $e-r \leq 1$, then clearly, $G$ is planar, so this completes the proof of the upper bound.

Let us observe that $r \geq n-1$ since every spanning tree is outerplanar, and that $r \geq n$ if $G$ is not a tree.

Theorem 2.4 is a slight improvement over the bounds derived in [5] for the degenerate crossing number. The lower bound is the same as the one in [5] but is given for $\operatorname{GCR}(G)$ instead for $\underline{\mathrm{CR}}(G)$. The upper bound improves the bound $\underline{\mathrm{CR}}(G) \leq e$ proved in [5].

\section{Degenerate and genus crossing number}

As we have observed earlier, the genus crossing number is always a lower bound for the degenerate crossing number. However, it is an open question if these two notions define different graph invariants. In fact, after several unsuccessful attempts to construct examples showing that the genus and the degenerate crossing number are distinct, we are inclined to propose the following

Conjecture 3.1. For every graph $G$, we have $\operatorname{GCR}(G)=\underline{\mathrm{CR}}(G)$.

Let us outline some thoughts related to this problem. Recall that the genus crossing number is related to the notion of having the graph embedded in a non-orientable surface $\mathbb{N}_{g}$ of genus $g$ and having a collection of $g$ pairwise disjoint 1-sided simple closed curves in $\mathbb{N}_{g}$ which yield an embedding in a planar surface (homeomorphic to the sphere with $g$ discs cut out) when the surface is cut along them. Such a collection of curves will be called a 
planarizing system of disjoint 1-sided curves, shortly a PDIS-system in $\mathbb{N}_{g}$. The following result is a simple consequence of the definitions.

Lemma 3.2. Let $G$ be a graph and $g$ be a positive integer. Then $\mathrm{CR}(G) \leq g$ if and only if $G$ has an embedding in the non-orientable surface $\mathbb{N}_{g}$ of genus $g$ and there exists a PD1Ssystem $\Gamma$ of curves in $\mathbb{N}_{g}$ such that every curve in $\Gamma$ intersects each edge of $G$ at most once.

This lemma may be of use when trying to settle Conjecture 3.1. Our next result shows that in order to prove Conjecture 3.1, it suffices to verify it for triangulations of nonorientable surfaces.

Proposition 3.3. Conjecture 3.1 is equivalent to the statement that for every positive integer $g$, every triangulation $G$ of the non-orientable surface $\mathbb{N}_{g}$ admits a PD1S-system $\Gamma$ of curves in $\mathbb{N}_{g}$ such that every curve in $\Gamma$ intersects each edge of $G$ at most once.

Proof. Clearly, if a triangulation $G$ would not satisfy the stated property, it would be a counterexample to Conjecture 3.1 .

Conversely, let $H$ be a counterexample to the conjecture and let $g=\tilde{\mathbf{g}}(H)<\underline{\mathrm{CR}}(H)$. Embed $H$ in $\mathbb{N}_{g}$ and and extend it to a triangulation $G$ of $\mathbb{N}_{g}$ (by adding edges and, if necessary, new vertices). Then $\operatorname{GCR}(G)=\tilde{\mathbf{g}}(G)=g$ and $\underline{\mathrm{CR}}(G) \geq \underline{\mathrm{CR}}(H)>g$.

It may be that a stronger conjecture holds. Recall that a pseudotriangulation of a surface $S$ is a multigraph (where we allow loops and multiple edges), which is 2-cell embedded in $S$ such that each facial walk has length three.

Conjecture 3.4. For every positive integer $g$, every loopless pseudotriangulation $G$ of $\mathbb{N}_{g}$ admits a PD1S-system $\Gamma$ of curves in $\mathbb{N}_{g}$ such that every curve in $\Gamma$ intersects each edge of $G$ at most once.

We do not even know of examples of pseudotriangulations with loops, where the only requirement is that loops are surface non-separating, for which Conjecture 3.4 would not hold. However, if we allow surface separating loops, counterexamples are easy to find.

\section{Minor and genus crossing number}

Bokal, Fijavž, and Mohar [1] introduced the notion of a crossing number, called the minor crossing number and denoted by $\operatorname{MCR}(G)$, which is minor-monotone: if a graph $H$ is a minor of a graph $G$, then $\operatorname{MCR}(H) \leq \operatorname{MCR}(G)$. This is achieved by defining the minor crossing number in the following way:

$$
\operatorname{MCR}(G)=\min \{\operatorname{CR}(H) \mid G \text { is a minor of } H\} .
$$

Theorem 2.1 implies that the genus crossing number is also minor-monotone.

Proposition 4.1. If a graph $H$ is a minor of a graph $G$, then $\operatorname{GCR}(H) \leq \operatorname{GCR}(G)$.

After having noted this relationship between the minor and the genus crossing number, it may be of interest to see to what extent are they related.

Proposition 4.2. For every graph $G$ we have $\operatorname{GCR}(G) \leq \operatorname{MCR}(G)$. 
Proof. Let $H$ be a graph such that $G$ is a minor of $H$ and $\operatorname{CR}(H)=\operatorname{MCR}(G)$. By replacing each crossing in an optimal drawing of $H$ by a crosscap, we see that $\tilde{\mathbf{g}}(H) \leq \mathrm{CR}(H)$. Since $G$ is a minor of $H$, we have $\tilde{\mathbf{g}}(G) \leq \tilde{\mathbf{g}}(H)$. Combining all these inequalities, we see that

$$
\operatorname{GCR}(G)=\tilde{\mathbf{g}}(G) \leq \tilde{\mathbf{g}}(H) \leq \mathrm{CR}(H) \leq \operatorname{MCR}(G),
$$

which we were to prove.

It can happen that the minor crossing number is much bigger than the genus crossing number. For example, it is proved in [1] that $\operatorname{MCR}\left(K_{n}\right) \geq\left\lceil\frac{1}{4}(n-3)(n-4)\right\rceil$. On the other hand, Corollary 2.2 shows that $\operatorname{GCR}\left(K_{n}\right)=\left\lceil\frac{1}{6}(n-3)(n-4)\right\rceil$. These results imply not only that MCR and GCR are distinct, but also that these crossing number parameters may be much different even for very sparse graphs. The following result gives further examples and some additional information.

Theorem 4.3. For every positive integer $n$ there is a graph $G$ of order $n$ such that $\operatorname{MCR}(G)-$ $\operatorname{GCR}(G) \geq \Omega\left(n^{2}\right)$. Moreover, if $n$ is even, there exists a cubic graph $H$ of order $n$ such that $\operatorname{CR}(H)=\operatorname{MCR}(H) \geq \operatorname{GCR}(H)+\Omega(n)$.

Proof. The first claim holds for $G=K_{n}$ as argued above. To prove the second claim, we shall assume that $n$ is of the form $n=m(m-3)$, where $m$ is a positive integer. The general case follows easily from this one, and the details are left to the reader.

Let us consider an embedding of the complete graph $K_{m}$ into the nonorientable surface of genus $g=\left\lceil\frac{1}{6}(m-3)(m-4)\right\rceil$. By expanding each vertex into a tree on $m-3$ vertices, we can obtain a cubic graph $H$ embedded in the same surface such that $K_{m}$ is a minor of $H$. This shows that the nonorientable genus of $H$ is equal to $g$. Since $H$ has $n=m(m-3)$ vertices, we conclude that $\operatorname{GCR}(H)=g=\frac{1}{6} n+O\left(n^{1 / 2}\right)$.

On the other hand, $\operatorname{MCR}(H)=\mathrm{CR}(H) \geq \operatorname{MCR}\left(K_{m}\right) \geq \frac{1}{4} n-O\left(n^{1 / 2}\right)$. Both inequalities show that $\operatorname{MCR}(H)-\operatorname{GCR}(H) \geq \frac{1}{12} n-O\left(n^{1 / 2}\right)$.

\section{References}

[1] D. Bokal, G. Fijavž and B. Mohar, The minor crossing number, SIAM J. Discrete Math. 20 (2006), 344-356.

[2] J. L. Gross, T. W. Tucker, Topological Graph Theory, Wiley - Interscience, New York, 1987.

[3] B. Mohar and C. Thomassen, Graphs on Surfaces, Johns Hopkins University Press, Baltimore, 2001.

[4] J. Pach and G. Tóth, Which crossing number is it anyway? J. Combin. Theory Ser. B 80 (2000), 225-246.

[5] J. Pach and G. Tóth, Degenerate crossing numbers, Discrete Comput. Geom. 41 (2009), 376384.

[6] L. Székely, A successful concept for measuring non-planarity of graphs: the crossing number, Discrete Math. 276 (2004), 331-352.

[7] C. Thomassen, The graph genus problem is NP-complete, J. Algorithms 10 (1989), 568-576.

[8] A. T. White, Graphs, Groups and Surfaces, North-Holland, 1973; Revised Edition: NorthHolland, 1984. 